\title{
Improving the Data Placement Algorithm of Randomization in SAN
}

\author{
Nianmin $\mathrm{Yao}^{1}, \mathrm{Jiwu}_{\mathrm{Shu}}^{2}$, and Weimin Zheng ${ }^{3}$ \\ 1 Department of computer science and technology, tsinghua university \\ lucos@126.com \\ 2 shujw@tsinghua.edu.cn \\ 3 zwm-dcs@tsinghua.edu.cn
}

\begin{abstract}
Using the randomization as the data placement algorithm has many advantages such as simple computation, long term load balancing, and little costs. Especially, some latest works have improved it to make it scale well while adding or deleting disks in large storage systems such as SAN (Storage Area Network). But it still has a shortcoming that it can not ensure load balancing in the short term when there are some very hot data blocks accessed frequently. This situation can often be met in Web environments. To solve the problem, based on the algorithm of randomization, an algorithm to select the hot-spot data blocks and a data placement scheme based on the algorithm are presented in this paper. The difference is that it redistributes a few very hot data blocks to make load balanced in any short time. Using this method, we only need to maintain a few blocks status information about their access frequency and more than that it is easy to implement and costs little. A simulation model is implemented to test the data placement methods of our new one and the one just using randomization. The real Web log is used to simulate the load and the results show that the new distributing method can make disks' load more balanced and get a performance increased by at most 100 percent. The new data placement algorithm will be more efficient in the storage system of a busy Web server.
\end{abstract}

\section{Introduction}

With the developing of the Internet, a dramatic growth of enterprise data storage capacity can be observed in the last couple of years. Many things including a lot of enterprise data coming onto the internet; data warehouse, e-business and especially the Web contents contribute the growth of storage. So the performance and capacity of the storage are becoming the bottleneck of IT's progress. Now, the SAN (Storage Area Network) is a popular technology to solve the problem. It can ensure the reliability, serviceability, scalability and availability of the storage. Though the SAN technology has so many virtues, there are also a few hard technical problems which need to be solved. One of these problems is how to place the data among the disks, e.g. the data placement algorithm. 
The good data placement algorithm can greatly improve the performance of the storage. More than that, it can also decrease the costs of reorganizing the data among the disks in SAN while adding or deleting a disk which is a very common operation in managing the storage system.

\section{Related Work}

An algorithm commonly being used to place data blocks is RAID [3]. It has been an industrial standard being applied in most of commercial products. This method has many virtues such as fault tolerance, high performance and so on. But it has a shortcoming that all the data must be reconstructed while adding or deleting disks. Additionally, the RAID 5 can not be used in large-scale storage system such as SAN because with the number of disks in the storage system increasing, its writing performance is becoming lower. So RAID is often used in one disk array and it should not be a good data placement algorithm in the whole SAN.

Another simple and effective method to distribute the data blocks among the SAN is using the randomization which is often used now. In this algorithm, every data block is put into a randomly selected physical disk block. But in fact, the physical disk block is not completely randomly selected. A pseudo-random function is often used because the right place for a data block can be easily found through a simple computation and need not to maintain a very large map list describing the relationship between the data blocks and the physical disk blocks. Randomly selecting the location of the data block is good at keeping the load balancing among all the disks in SAN in a long run. But as the RAID technology, while adding or deleting the disks, the whole data must be reorganized. And more than that, it can't ensure that load is balanced in any short term, for example, when there are hot data accessed frequently during a short time.

To decrease the costs of reorganizing the data when adding or deleting disks, an improved randomization algorithm was presented in 2]. The main work of [2] focused on the hash function being used in the randomization algorithm. Once the number of disks is changed, it only needs to change the hash function and move a little data in order to reconstruct the data. Similar works have been done in $[1,9]$.

Being improved, the algorithm of randomization now has so many advantages such as simple computation, long term load balancing, little costs and high scalability. But all the methods described above have the same shortcoming that they all can not ensure load is balanced when there is hot data during some short time. Because if there are some data blocks which are accessed very frequently, then the load is more often focused on a few disks where these hot data blocks are saved. In this situation which will often be met in Web environment, the load is not balanced and the storage resources can not be fully utilized while using the algorithms discussed above to distribute the data blocks. Intuitively, if the information of the access frequency of the data blocks is applied into the distributing scheme, we can make disks loads more balanced. But all the 
work above did not use this kind of information, because it will cost a lot of resources if the information of all the data blocks access frequencies are to be maintained. Some data placement algorithms which take the access frequency of blocks into account have been given, for example [5] , but they can only be used in a small storage system such as a disk array, because they totally ignore the high costs of maintaining all the information of access frequency of blocks. The data placement algorithm must be simple and effective enough to be used. We will give a new method based on the randomization in this paper to distribute the data blocks which can keep short term load balancing and costs little.

\section{The Algorithm to Select Hot Data Blocks}

The network traffic follows the self-similarity model, e.g. it can be bursty on many or all time scales 6 . So, there will often be a few hot spot data blocks which burden most loads in the servers. As discussed, the above distributing methods obviously can not deal with this situation well and make full use of the storage resources. Because not until the information of data blocks access frequency is concerned in distributing data blocks, we can not really keep the load balancing. But how to decrease the costs of maintaining the information of all the data blocks access frequency? In fact, for some servers on the Internet, we only need to maintain the access frequency of a small part of all the data blocks, because there are only a small part of files of the servers on the Internet which can be accessed frequently during a short time. In the study of distributed Web servers, many works make use of this characteristic of servers [2, 3]. For example, study of the world cup 98 s Web log indicates that only $3 \mathrm{MB}$ space covers $99 \%$ requests in some short time. So the critical point to solve the problem is how to effectively select these hot data blocks.

In this section, we will give an algorithm to select the hot data blocks. Tts data structures are two lists which have fixed length. One called "HL" which will hold the hot data blocks and the other called "CL" which contains candidate hot data blocks. Their length is decided by the storage space and it can be $1 \%$ of the whole space in blocks or lower. HL's elements are ordered by blocks' access frequency and CL's elements are ordered approximately by their insert sequence. The elements' data structure contained in the list has information about address or access frequency such as frequency and hot_levle. When we say $D 1>D 2$, it means that D1.hot_levle $>$ D2.hot_level or else D1.hot_levle $=$ D2.hot_level and D1.frequency $>$ D2.frequency.

The algorithm is described below. In the algorithm, "HOT_LEVEL", "UPGRADE_LEVEL" and "CYCLE" are the predefined parameters. CYCLE is the interval of the number of time units to check and organize the two lists. "Time" always denotes the current time. The text behind "//" is comment.

(Step 1) Old_time=time;

make all data blocks' frequency in HL and CL be 0 ;

(Step 2) A request of data block D comes; 


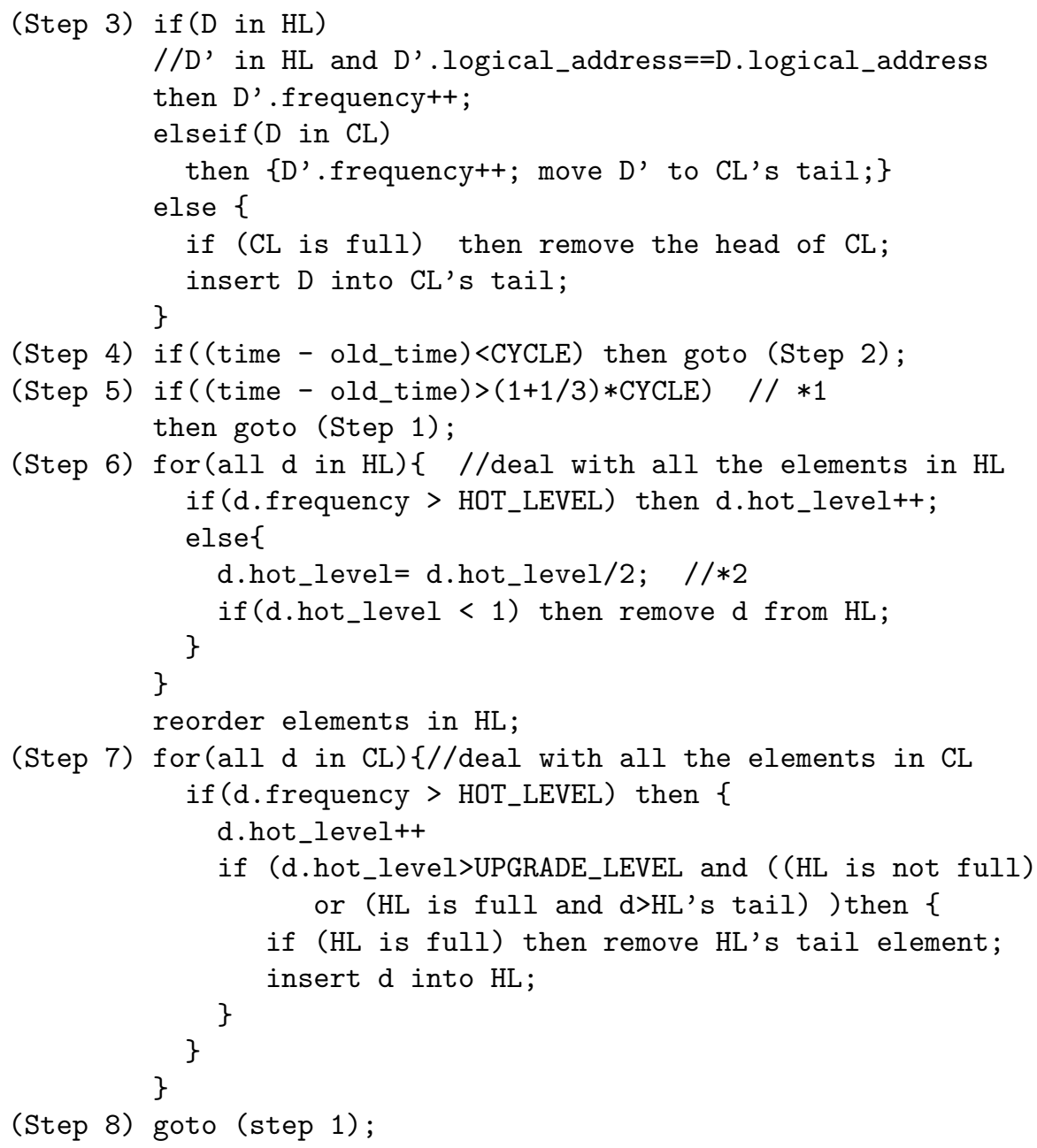

We can see that when the algorithm is functioning, the elements in HL are the hottest data blocks whose access count is more than HOT_LEVEL in about a time unit for several continuous CYCLEs. The sentence marked by "* 1 ” ensure that the computing error of frequency is not too big and when this sentence is true, we can believe that the system is much idle because there is no request in $1 / 3$ (can be changed according to the status of load) time unit. The sentence marked by " 2 " makes the data block's hot_level decrease more quickly than it increases. Each time when an element in CL is accessed, it will be moved to the tail of CL so that hot data blocks are not easy to be squeezed out of the CL because we always squeeze the head element out of the CL. So when running for a while, elements in the CL must be relatively hot and can be candidates to be selected into the HL.

Obviously, this algorithm can make sure that all the hottest data blocks will be put in the HL and its costs are very low because there are only a little simple 
computations in it and it needs not to access the disks and maintain all the blocks access frequencies.

\section{A New Data Placement Scheme}

Now, we can get the information about the hottest data blocks by the algorithm given in section 3, then the next question is how to use it. We will present a new data placement scheme in this section based on the algorithm of randomization and the algorithm to select the hottest data blocks we have given.

SAN systems usually provide storage virtualization functions. Its architecture is described in fig. 1. The meta data server maintains all the information about the data distribution. Before a server reads a data block, it must first get the real location of the data from the meta data server. So we can implement the function of distributing data blocks in the meta server. By the way, some meta data can be cached in the server and the actual data accesses need not pass through the meta data server, so the meta data server usually can not become the bottleneck of the system.

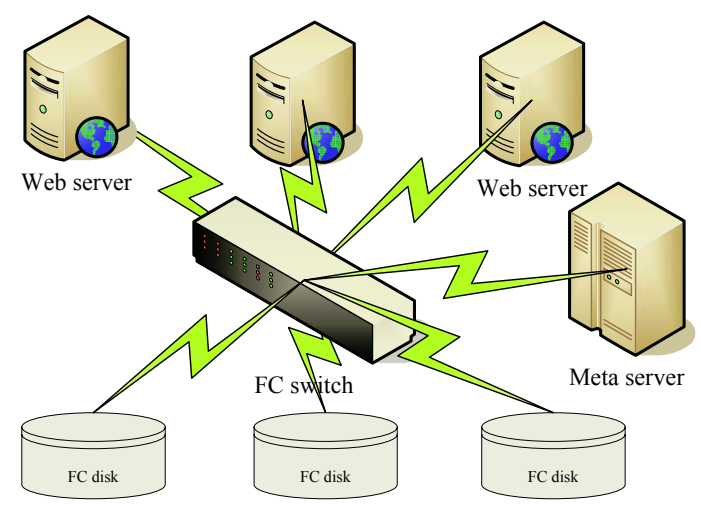

Fig. 1. Virtual Storage Architecture in SAN

In the meta server, we can implement the data placement function like this:

We always assign MAXQL as the command queue length of the busiest disk, MINQL as the command queue length of the idlest disk. In the following description, MAX_QUEUE_LENGTH and DIFF_QUEUE_LENGTH are pre-defined parameters. Initially, the data placement follows the randomization algorithm, for example, using some pseudo-random hash function. When the system is running, how our data placement scheme is functioning can be described below. These steps can be inserted after the algorithm's (Step 7) and before its (Step 8) described in section 3 . 
(Step 1) Get the correct value of MAXQL and MINQL.

(Step 2) if not (MAXQL> MAX_QUEUE_LENGTH) then goto (Step 9);

(Step 3) if not (MAXQL-MINQL) >DIFF_QUEUE_LEN then goto (Step 9);

(Step 4) if (There aren't any hot spot data blocks in the most busy disk)

then goto (Step 9);

(Step 5) Copy the most hot block to the most idle disk;

(Step 6) Change the physical address of the moved data block in meta server;

(Step 7) if (There aren't any data blocks whose physical addresses were changed and now are not hot) then goto (Step 9);

(Step 8) Delete the redundant copies of the ever hot data blocks and change their physical addresses to their original addresses in meta server;

(Step 9) end;

In the scheme, we do not delete the original moved data block, and then the hot data block has two redundant copies, so we can also schedule a few requests to the hot data blocks for better balancing the load. In the scheme, some disks' space is spent for the redundant copy of hot data blocks. But it is so small that it can be ignored. For example, if the scheme is used in the Web server of World Cup 98, the wasted disk space is no more than 3M bytes.

In this distributing scheme, most of the data blocks' placement follows the randomization method. It only needs to process a little hot spot data blocks which have much effect on the storage performance during a short time. So, its costs are low enough to be applied in the practical storage system.

\section{$5 \quad$ Experiments}

To prove our design's efficiency, we implemented a simulation model depicted in fig. 1] using the CSIM18 10] which is a famous tool to simulate the discrete events. The model we are using is similar to the one described in [2]. In the model, one "ROUND" is defined as the time needed by a disk to process a data block request. The servers continuously send requests to the disks and record their response time. The difference between our model and the one in [2] is that in our model the disks' requests queue is long enough to hold all the requests, e.g. the requests will not be discarded. Especially, we use the real Web access log to drive the model, so it can truly reflect the SAN environment. The 1000,000 requests we used to drive the model are extracted from the world cup 98's web $\log$ at 1998.5.1. For simplification, every data block request represents a file request in the log.

In the first experiment, we set the number of disks as 5 and change the arriving rate of the requests. Its test results are drawn in fig. 2, In fig. 2, the $\mathrm{X}$ axis represents the interval of incoming requests in percentile of ROUND. So with the number decreasing, the load is heavier. We can see that in most 
of the situations, our distributing method is much better than the one using randomization except that when system is too idle or too busy. We deem that in the two extreme situations, the load balancing function of the new distributing method doesn't work well. The reason of the phenomena can be gotton in our data placement method such as (Step 5) in section 3 and (Step 2), (Step 3) in section 4 . Because when the storage system are too idle or busy, it will not need to balance the load what is just our data placement method does.

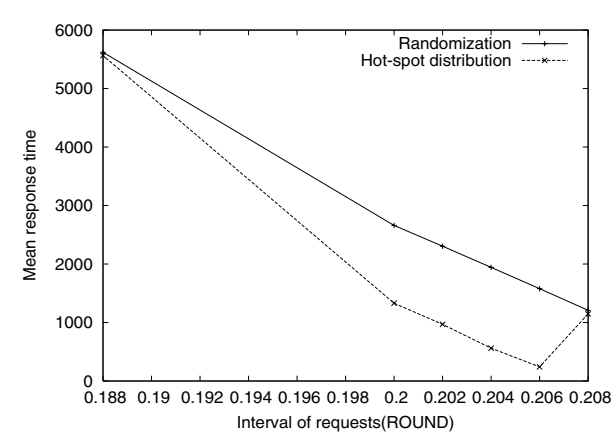

Fig. 2. Tests results of two data placement methods

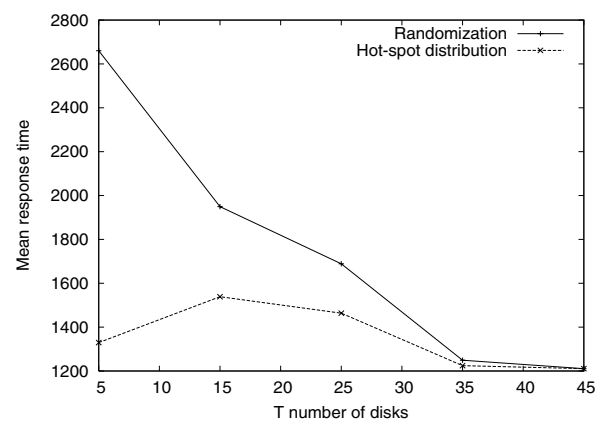

Fig. 3. Test results while changing the number of disks

In the testing, we found that the parameters of the model especially the HOT_LEVEL have very big effect on the performance. For example, when we set the HOT_LEVEL as 8, then when the interval of requests is $20 \%$ of a ROUND, the mean response time can reach at 880 ROUNDs which is much better than before. So if the parameters of the model can be adjusted dynamically according to the load of the system, we can get more performance enhancements. This will be our future work.

In our second experiment, we change the number of disks in the model and get the results described in fig. 3. In order to let the system be not too idle, we set the interval of requests as $1 / \mathrm{n}$ ROUND, in which $\mathrm{n}$ denotes the number of disks. Obviously, we can see from fig. 3 that our data placement scheme is much better than the other. It can be noticed that when the number of disks is 45 , the two methods get the same results. We believe that it is because that the system is too idle in this configuration. The followed test proved our conjecure that when we increase the load, we get much better results than the randomization method. For simplification, we would not show this results here.

\section{Conclusions}

In this paper, some data placement algorithms are analyzed and they all can not keep disks' load balanced when there are hot data accessed frequently which have much effect on the performance of the system. The reason is that they do 
not take the access frequency of disks into consideration because they may think it will cost too much. So we present a new data placement method based on the randomization algorithm which can evenly distributing the hottest data blocks among disks and is also very easy to implement and costs little.

We implement a simulation model to test the new data placement algorithm and it proves that it can better balance the load of disks and get much higher performance. We will implement it in a true SAN system with the virtual storage function in the future. We can say that it will be very promising while applying the data placement algorithm into the storage system of a busy Web server.

Acknowledgements. This research is supported by China Postdoctor foundation(No.023240008), the National Natural Science Foundation of China (No: 60473101) and the National High-Tech Research and Develop Program of China (No: 2004AA111120).

\section{References}

1. A. Brickmann, K. Salzwedel, and C. Scheideler: Compact, adaptive placement schemes for non-uniform capacities. In Proceedings of the 14th ACM Symposium on Parallel Algorithms and Architectures (SPAA), Winnipeg, Manitoba, Canada (Aug, 2002) 53-62

2. Cherkasova L, Karlsson M: Scalable web server cluster design with workload-aware request distribution strategy with WARD. In: Proceedings of the 3rd International Workshop on Advanced Issues of E-Commerce and Web-Based Information Systems. Los Alamitos: IEEE Computer Society Press (2001) 212-221

3. D. A. Patterson, G. Gibson and R. H. Katz: A case for Redundant Arrays of Inexpensive Disks(RAID). In Proceedings of the 1988 ACM Conference on Management of Data(SIGMOD) (June 1988) 109-116

4. Du ZK, Zheng MY, Ju JB: A distributed algorithm for content-aware Web server clusters. Journal of Software, 2003,14(12) 2068-2073

5. G. Weikum, P. Zabback, and P. Scheuermann: Dynamic File Allocation in Disk Arrays. In Proc. of ACM SIGMOD, (May 1991) 406-415

6. J. C. Mogul: Network behavior of a busy web server and its clients, Technical Report WRL 95/5, DEC Western Research Laboratory, Palo Alto, CA (1995)

7. M. E. Crovella and A. Bestavros: Self-similarity in world wide web traffic: Evidence and possible causes, In Proceedings of the 1996 ACM SIGMETRICS International Conference on Measurement and Modeling of Computer Systems (May 1996)

8. P. Berenbrink, A. Brinkmann, and C. Scheideler: Design of the PRESTO multimedia storage network. In International Workshop on Communication and Data Management in Large Networks, Paderborn, Germany (October 5 1999)

9. R. J. Honicky, E. L. Miller: A fast algorithm for online placement and reorganization of replicated data. 17th International Parallel and Distributed Processing Symposium (IPDPS) (2003)

10. Schwetman, H.: Object-oriented simulation modeling with C++/CSIM17. In Proceedings of the 1995 Winter Simulation Conference. ed. C. Alexopoulos, K. Kang, W. Lilegdon, D. Goldsman. Washington, D.C. (1995) 529-533 